\title{
Influência do nitrato e fosfato no crescimento de Schizomeris leibleinii Kützing (Chaetophorales, Chlorophyta)
}

\author{
Jeferson Luizi Pereira ${ }^{1,2}$ e Luis Henrique Z. Branco ${ }^{1}$
}

Recebido em 23/01/2006. Aceito em 24/08/2006

\begin{abstract}
RESUMO - (Influência do nitrato e fosfato no crescimento de Schizomeris leibleinii Kützing (Chaetophorales, Chlorophyta)). Schizomeris leibleinii Kützing é uma alga filamentosa comumente encontrada em ambientes aquáticos continentais eutrofizados ou poluídos por nutrientes orgânicos e é um potencial organismo indicador de poluição orgânica. Estudos envolvendo o cultivo de $S$. leibleinii sob concentrações variáveis de nitrato e fosfato foram efetuados durante períodos de 50 dias. A biomassa foi expressa através da quantificação da concentração de clorofila $a$ relativa a diferentes tempos de cultivo. Além da análise das curvas de crescimento, os dados obtidos foram submetidos à Anova (uma via) e teste de Tukey para comparação entre os tratamentos. Os resultados revelaram que S. leibleinii apresentou crescimento rápido sob baixas concentrações de nitrato e fosfato, mas o melhor desempenho ocorreu em concentrações altas de nitrato. Verificou-se nesta espécie que condições de estresse ambiental provocam, inicialmente, crescimento rápido, possivelmente acompanhado de intensa reprodução. Apesar do rápido desenvolvimento, a avaliação das curvas de crescimento de $S$. leibleinii sugere que esta espécie é um estrategista em $k$. Além disso, a alga mostrou-se mais dependente da concentração de nitrato do que de fosfato, indicando o nitrogênio como limitante do crescimento em meio de cultura.
\end{abstract}

Palavras-chave: crescimento, fosfato, nitrato, Schizomeris leibleinii, poluição

\begin{abstract}
Influence of nitrate and phosphate on the growth of Schizomeris leibleinii Kützing (Chaetophorales, Chlorophyta)). Schizomeris leibleinii Kützing is an algal species commonly found in eutrophic freshwater or in water polluted by organic nutrients and shows high potential as an organic pollution indicator. S. leibleinii was grown at different nitrate and phosphate concentrations for 50 days. Biomass was determined by quantifying chlorophyll $a$ concentrations for different cultivation periods. Besides growth-curve analysis, data were evaluated by ANOVA (one way) and by Tukey's test for comparing treatments. The study revealed that S. leibleinii grew rapidly at low concentrations of nitrate and phosphate, but the best development occurred at high nitrate concentrations. Environmental stress initially promoted fast growth in this species, possibly accompanied by intense reproduction. In spite of the rapid growth of $S$. leibleinii, growth curves suggested that this species is probably a $k$-strategist. In addition, the alga was more dependent on nitrate than on phosphate concentration, indicating nitrogen as the limiting nutrient of growth in culture.
\end{abstract}

Key words: growth, nitrate, phosphate, Schizomeris leibleinii, pollution

\section{Introdução}

Ambientes lóticos, bem como todos os ecossistemas aquáticos, freqüentemente estão sujeitos aos mais variados tipos de perturbações. Segundo $\mathrm{Pal}$ et al. (1992), o despejo de substâncias orgânicas no ambiente, resultantes de atividades domésticas, agrícolas e industriais, caracteriza a poluição orgânica. A entrada destas substâncias, bem como de outras contendo nutrientes, pode inicialmente promover a eutrofização, que contribui para um grande desenvolvimento algal e que, na maioria das vezes, provoca transtornos dos mais variados tipos como entupimento de canos, odor e sabor desagradáveis na água, problemas de saúde através da liberação de substâncias nocivas ao homem, além da depreciação visual do corpo d'água (De Vries et al. 1985; Pal et al. 1992).

Análises utilizando algas são ferramentas valiosas para monitorar a eutrofização de ambientes aquáticos e para quantificar a resposta biológica às mudanças nas concentrações de nutrientes (Maloney \& Miller 1975; Biggs 1989; McCormick \& Cairns 1994; Necchi Júnior et al. 1994; Kelly \& Whitton 1998; Branco \& Pereira 2002). Algas verdes filamentosas, principalmente da ordem Chaetophorales, são freqüentemente abundantes em águas rasas e eutrofizadas (Hillebrand 1983; Klapwijk et al. 1983;

\footnotetext{
1 Universidade Estadual Paulista, Departamento de Zoologia e Botânica, Rua Cristóvão Colombo 2265, 15054-000 São José do Rio Preto, SP, Brasil

2 Autor para correspondência: j.luizi@terra.com.br
} 
Necchi Júnior et al. 1994; Branco \& Pereira 2002). Contudo, estudos envolvendo suas respostas biológicas às alterações das concentrações de nutrientes são escassos.

Comumente, espécies do gênero Stigeoclonium Kützing (Chaetophorales) são escolhidas como organismos teste nestes estudos, devido à sua comum ocorrência durante o ano todo no perifíton de águas rasas e geralmente eutrofizadas (Francke \& Ten Cate 1980; De Vries et al. 1983; De Vries \& Hotting 1985; De Vries et al. 1985). Com a finalidade de explicar a distribuição de Stigeoclonium de acordo com os níveis de nutrientes em seus hábitats, Francke \& Ten Cate (1980) avaliaram as respostas do crescimento de várias linhagens deste organismo aumentando os níveis de fosfato e amônio em cultura. De Vries et al. (1983) testaram, como organismos de bioensaio, 11 linhagens do gênero Stigeoclonium para quantificar as concentrações de nitrogênio e fósforo limitantes ao crescimento. De Vries et al. (1985) analisaram os efeitos das concentrações combinadas de nitrato e fosfato na produtividade de várias linhagens de Stigeoclonium, em meio sintético e em água do próprio ambiente, observando que o aumento da produtividade algal após a adição de um nutriente depende da concentração do outro. Ainda De Vries \& Hotting (1985) testaram Stigeoclonium tenue (Agardh) Kützing como organismo de bioensaio em águas recebendo efluentes de duas estações de tratamento de esgoto, contendo taxas variadas de fosfato $\left(\mathrm{PO}_{4}-\mathrm{P}\right)$ e amônio $\left(\mathrm{NH}_{4}-\mathrm{N}\right)$, sugerindo que a presença das águas residuais inibia o crescimento desta alga devido, possivelmente, ao amônio ou a metais presentes na mesma.

A alga Schizomeris leibleinii Kützing é um organismo comum em ambientes aquáticos tropicais eutrofizados ou impactados por poluição orgânica (Campbell \& Sarafis 1972; Sarma \& Chaudhary 1975; Yadava \& Pandey 1980; Necchi Júnior et al. 1994; Branco \& Pereira 2002) ou industrial (Tiwari 1994). Alguns trabalhos sugerem que $S$. leibleinii seja indicador de poluição, merecendo atenção especial já que se desenvolve bem em ambientes fortemente eutrofizados ou organicamente poluídos (Necchi Júnior et al. 1994; Branco \& Pereira 2002). Dessa maneira, o conhecimento de sua biologia e de sua capacidade de tolerância às condições ambientais torna-se importante para projetos relacionados ao biomonitoramento ou indicação biológica dos níveis de nutrientes orgânicos.

O presente trabalho tem por objetivo estudar o desenvolvimento de S. leibleinii em cultura através da variação das concentrações dos nutrientes nitrogênio e fósforo, nas formas de nitrato e fosfato, avaliando o crescimento da espécie em relação a estes fatores.

\section{Material e métodos}

Os espécimes de Schizomeris leibleinii Kützing utilizados para desenvolver os experimentos foram coletados no Ribeirão Claro $\left(20^{\circ} 46^{\prime} 33^{\prime \prime} \mathrm{S}\right.$ e 49¹1'49”'W) no município de Guapiaçu, Estado de São Paulo. Amostras do crescimento algal foram coletadas e acondicionadas em frascos de vidro com água do próprio local e levadas ao laboratório para limpeza e obtenção de cultura unialgal (não axênica) desta espécie.

Para a obtenção da cultura unialgal, o material vivo foi lavado várias vezes em tubos de ensaio com água deionizada esterilizada. Em seguida, três ou quatro filamentos de $S$. leibleinii foram transferidos para erlenmeyers contendo meio básico de Bold (MBB) líquido (Nichols 1973). Os frascos com os inóculos foram mantidos em câmaras de cultivo com fotoperíodo de $12 \mathrm{~h}$ claro/ $12 \mathrm{~h}$ escuro, sob intensidade luminosa de $100 \mu \mathrm{mol} \mathrm{m} \mathrm{m}^{-2} \mathrm{~s}^{-1}\left( \pm 2,0 \mu \mathrm{mol} \mathrm{m} \mathrm{m}^{-2} \mathrm{~s}^{-1}\right)$ e dois valores de temperatura 15 e $20^{\circ} \mathrm{C}$ (modificado de Sarma \& Chaudhary 1975; Yadava \& Pandey 1980).

Os cultivos unialgais utilizados nos experimentos de variação de meio, provêm de duas subamostras de uma mesma cepa de $S$. leibleinii que foram mantidos inicialmente em MBB desprovido de nitrato ou fosfato, por um período de cinco dias, para garantir que as algas esgotassem suas reservas internas e absorvessem os nutrientes em questão diretamente do meio de cultura (De Vries \& Hotting 1985).

Tomando-se MBB como solução padrão, as concentrações de nitrato e fosfato do meio foram modificadas isoladamente (apenas nitrato ou fosfato) ou em conjunto (os dois nutrientes), obtendo-se concentrações aproximadamente cinco vezes superior e dez vezes inferior à concentração padrão destes nutrientes em MBB (Tab. 1). Foram utilizadas 25 réplicas para o controle $(\mathrm{MBB})$ e para cada tratamento $(\mathrm{MBB}+\mathrm{N}, \mathrm{MBB}-\mathrm{N}, \mathrm{MBB}+\mathrm{P}, \mathrm{MBB}-\mathrm{P}, \mathrm{MBB}+\mathrm{N} / \mathrm{P}$ e MBB-N/P). Em cada frasco foi inoculado um grupo de filamentos de $S$. leibleinii, totalizando uma biomassa inicial de $5 \mathrm{mg}$ da alga em $75 \mathrm{~mL}$ das variações do meio de cultura MBB citadas. Após o inóculo, os frascos foram levados para câmaras de cultivo e ficaram expostos a um valor de luminosidade de $100 \mu \mathrm{mol} \mathrm{m}^{-2} \mathrm{~s}^{-1}\left( \pm 2,0 \mu \mathrm{mol} \mathrm{m} \mathrm{s}^{-2}\right)$ por um período de 50 dias, sob fotoperíodo de $12 \mathrm{~h}$ claro/12 $\mathrm{h}$ escuro e 
Tabela 1. Concentrações de nitrato e fosfato no Meio Básico de Bold (controle) e demais tratamentos utilizados durante os experimentos.

\begin{tabular}{ccr}
\hline \multirow{2}{*}{ Meios de cultura } & \multicolumn{2}{c}{ Concentrações } \\
\cline { 2 - 3 } & \multicolumn{1}{c}{$\mathrm{PO}_{4}^{+}$} & \multicolumn{1}{c}{$\mathrm{NO}_{3}^{-}$} \\
\hline MBB & $1,720 \mathrm{mM}$ & $2,940 \mathrm{mM}$ \\
MBB-N & $1,720 \mathrm{mM}$ & $0,294 \mathrm{mM}$ \\
MBB+N & $1,720 \mathrm{mM}$ & $14,470 \mathrm{mM}$ \\
MBB-P & $0,172 \mathrm{mM}$ & $2,940 \mathrm{mM}$ \\
MBB+P & $8,600 \mathrm{mM}$ & $2,940 \mathrm{mM}$ \\
MBB-N/P & $0,172 \mathrm{mM}$ & $0,294 \mathrm{mM}$ \\
MBB+N/P & $8,600 \mathrm{mM}$ & $14,470 \mathrm{mM}$ \\
\hline
\end{tabular}

temperatura de $20^{\circ} \mathrm{C}\left( \pm 1^{\circ} \mathrm{C}\right)$, já que esta temperatura estava mais próxima do ambiente natural desta alga.

A cada 10 dias, cinco frascos de cada tratamento foram retirados das câmaras de cultivo e submetidos à raspagem com bastões de polietileno e espátulas de silicone para o desprendimento dos filamentos algais que cresciam sobre o fundo e paredes laterais do mesmo. O meio de cultura com o material em suspensão foi filtrado utilizando-se filtros de microfibra de vidro Whatman $\mathrm{GF} / \mathrm{C}$ (retenção de partículas $1,2 \mu \mathrm{m})$. Devido à clorofila $a$ ser o pigmento fotossintetizante dominante e o mais abundante nas algas, a partir deste material, a biomassa de cada amostra foi avaliada com base na quantificação de clorofila $a$ pelo método espectrofotométrico de acordo com Wetzel \& Westlake (1971) e Wetzel \& Likens (1990).

Para realização das análises estatísticas utilizouse o programa estatístico Minitab versão 10.1. Através do teste de Shapiro-Wilk's (W) (Zar 1999), foi constatada a distribuição normal dos dados de biomassa das culturas estudadas, garantindo a aplicabilidade de testes paramétricos de Análise de variância (Anova - uma via) e teste de Tukey $(\mathrm{p}<0,05)$, para a avaliação de possíveis diferenças quanto aos tratamentos aplicados durante o experimento.

\section{Resultados}

Nos experimentos referentes à variação de nitrato e fosfato isoladamente (Fig. 1A-E), a produção máxima de biomassa ocorreu sempre no $40^{\circ}$ dia, com exceção apenas do material cultivado em MBB-N, cuja produtividade máxima ocorreu no $20^{\circ}$ dia de cultivo. A Anova realizada com base nos dados do experimento de variação de nitrato (Tab. 2) revelou diferenças significativas no $10^{\circ}(\mathrm{F}=9,45 ; \mathrm{p}<0,00), 30^{\circ}(\mathrm{F}=5,11$; $\mathrm{p}<0,05), 40^{\circ}(\mathrm{F}=29,64 ; \mathrm{p}<0,00)$ e $50^{\circ}(\mathrm{F}=60,90$; $\mathrm{p}<0,00)$ dias de crescimento quando comparadas aos cultivos MBB (controle). O teste de Tukey demonstrou que no $10^{\circ}(\mathrm{dms}=3,81), 30^{\circ}(\mathrm{dms}=9,20), 40^{\circ}$ $(\mathrm{dms}=17,84)$ e $50^{\circ}(\mathrm{dms}=6,85)$ dias, a produção de clorofila $a$ em MBB foi significativamente maior que a produção em MBB-N, enquanto no $30^{\circ}(\mathrm{dms}=9,20)$ e $50^{\circ}(\mathrm{dms}=6,85)$ dias esta diferença ocorreu apenas entre a produção em $\mathrm{MBB}$ e $\mathrm{MBB}+\mathrm{N}$. No entanto, no $10^{\circ}(\mathrm{dms}=3,81)$, o crescimento foi mais acentuado em MBB-N que $\mathrm{MBB}+\mathrm{N}$, enquanto nos $40^{\circ}$ $(\mathrm{dms}=17,84)$ e $50^{\circ}(\mathrm{dms}=6,85)$ dias ocorreu o contrário; o crescimento observado foi menor em MBB-N do que em MBB $+N$. Os dados do experimento de variação de fosfato (Tab. 2) quando submetidos à Anova mostraram que no $10^{\circ}(\mathrm{F}=16,24$; $\mathrm{p}<0,00), 40^{\circ}(\mathrm{F}=17,68 ; \mathrm{p}<0,00)$ e $50^{\circ}(\mathrm{F}=10,06$; $\mathrm{p}<0,00)$ dias de crescimento houve diferenças significativas entre os tratamentos. O teste de Tukey demonstrou que no $10^{\circ}(\mathrm{dms}=5,23)$ dia de crescimento a produção de clorofila $a$ em MBB foi menor em relação ao tratamento MBB-P, no entanto, no $40^{\circ}$ $(\mathrm{dms}=16,65)$ e $50^{\circ}(\mathrm{dms}=10,66)$ dias o crescimento foi maior em MBB foi maior que em MBB-P. O teste de Tukey mostrou ainda que o crescimento algal do tratamento MBB-P foi maior que em $\mathrm{MBB}+\mathrm{P}$ apenas no $10^{\circ}$ dia $(\mathrm{dms}=5,23)$. Nos $40^{\circ}(\mathrm{dms}=16,65)$ e $50^{\circ}$ $(\mathrm{dms}=10,66)$ dias de crescimento este mesmo teste mostrou que entre $\mathrm{MBB}$ e $\mathrm{MBB}+\mathrm{P}$ ocorreram diferenças significativas quanto às médias obtidas em relação à produção de clorofila $a$, sento esta última maior em MBB que em $\mathrm{MBB}+\mathrm{P}$.

Nos tratamentos envolvendo as combinações de nitrato e fosfato (Fig. 1F-G) ocorreu uma inversão quanto aos valores máximos e mínimos. Em MBB+N/P, maior concentração ocorreu no $50^{\circ}$ dia, enquanto em MBB-N/P, neste mesmo período, obteve-se a menor concentração de clorofila $a$. A Anova envolvendo os dados deste tratamento (Tab. 2) revelou que em todos os períodos de crescimento houve diferenças significativas: $10^{\circ}(\mathrm{F}=17,11 ; \mathrm{p}<0,00), 20^{\circ}(\mathrm{F}=12,21$; $\mathrm{p}<0,001), 30^{\circ}(\mathrm{F}=12,71 ; \mathrm{p}<0,001), 40^{\circ}(\mathrm{F}=42,03$; $\mathrm{p}<0,00)$ e $50^{\circ}$ dias $(\mathrm{F}=59,30 ; \mathrm{p}<0,00)$. O teste de Tukey mostrou que o crescimento algal medido quanto à produção de clorofila $a$ foi nitidamente maior em MBB que em MBB-N/P no $30^{\circ}(\mathrm{dms}=9,55), 40^{\circ}$ $(\mathrm{dms}=16,49)$ e $50^{\circ}(\mathrm{dms}=7,03)$ dias; enquanto entre $\mathrm{MBB}$ e $\mathrm{MBB}+\mathrm{N} / \mathrm{P}$ esta mesma diferença quanto à produção de clorofila $a$ ocorreu durante todo o período de incubação. Já entre os tratamentos MBB-N/P e 
A
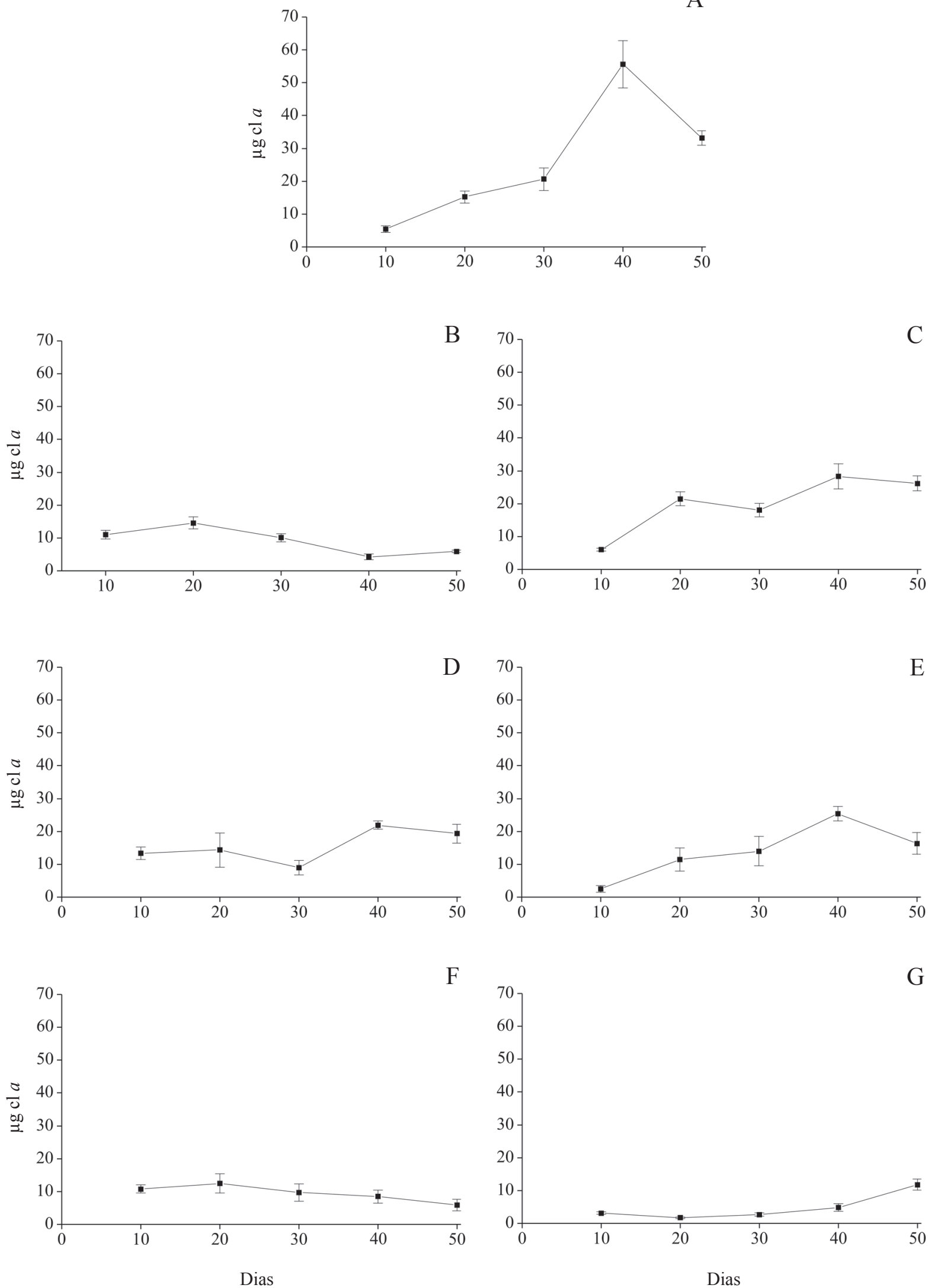

Figura 1. Crescimento de Schizomeris leibleinii Kützing durante 50 dias em diferentes concentrações de nitrato e fosfato. (A) MBB (controle), (B) MBB-N, (C) MBB+N, (D) MBB-P, (E) MBB+P, (F) MBB-N/P, (G) MBB+N/P. 
Tabela 2. Valores de clorofila $a$ em $\mu \mathrm{g} \mathrm{mL}{ }^{-1}$ por tratamento (média $\pm \mathrm{ep} ; \mathrm{n}=5,{ }^{*} \mathrm{n}=4$ ) expressando o crescimento de Schizomeris leibleinii Kützing por 50 dias em diferentes concentrações de nitrato e fosfato. (ep = erro padrão).

\begin{tabular}{|c|c|c|c|c|c|}
\hline \multirow[t]{2}{*}{ Meios de cultura } & \multicolumn{5}{|c|}{ Dias de crescimento } \\
\hline & 10 & 20 & 30 & 40 & 50 \\
\hline $\mathrm{MBB}$ & $5,38 \pm 1,04$ & $15,16 \pm 1,84$ & $20,64 \pm 3,50$ & $55,66 \pm 7,22$ & $33,18 \pm 2,14$ \\
\hline MBB-N & $11,04 \pm 1,33$ & $14,57 \pm 1,83$ & $10,06 \pm 1,20$ & $4,22 \pm 0,91$ & $5,90 \pm 0,35$ \\
\hline $\mathrm{MBB}+\mathrm{N}$ & $6,04 \pm 0,43$ & $21,44 \pm 2,13$ & $18,09 \pm 2,04$ & $28,32 \pm 3,76$ & $26,18 \pm 2,28$ \\
\hline MBB-P & $13,29 \pm 1,91$ & $14,37 \pm 5,20^{*}$ & $8,90 \pm 2,19$ & $21,91 \pm 1,25$ & $19,36 \pm 2,87$ \\
\hline $\mathrm{MBB}+\mathrm{P}$ & $2,49 \pm 1,03$ & $11,46 \pm 3,47$ & $13,99 \pm 4,48$ & $25,37 \pm 2,21$ & $16,36 \pm 3,35$ \\
\hline MBB-N/P & $10,75 \pm 1,22$ & $12,49 \pm 2,99$ & $9,65 \pm 2,61$ & $8,44 \pm 1,95$ & $5,84 \pm 1,77$ \\
\hline $\mathrm{MBB}+\mathrm{N} / \mathrm{P}$ & $3,12 \pm 0,36$ & $1,74 \pm 0,22$ & $2,71 \pm 0,47$ & $4,85 \pm 1,22$ & $11,79 \pm 1,65$ \\
\hline
\end{tabular}

$\mathrm{MBB}+\mathrm{N} / \mathrm{P}$ o crescimento algal foi maior em $\mathrm{MBB}+\mathrm{N} / \mathrm{P}$ que em MBB-NP apenas no $10^{\circ}$ $(\mathrm{dms}=3,57)$ e $20^{\circ}(\mathrm{dms}=7,67)$ dias de crescimento.

Ao final de 50 dias de crescimento a produção de clorofila $a$ em MBB foi maior do que a produtividade observada nos demais tratamentos (Tab. 3). No meio de cultura com maior concentração de nitrato $(\mathrm{MBB}+\mathrm{N})$ a resposta de crescimento algal esteve próxima da obtida em $\mathrm{MBB}$, enquanto em meio contendo mais fosfato $(\mathrm{MBB}+\mathrm{P})$, o crescimento foi quase a metade de MBB (Fig. 2). A biomassa de Schizomeris leibleinii Kützing nos tratamentos com concentrações de nitrato e fosfato inferiores ao controle (MBB-N e MBB-P) foi baixa apenas em relação ao nitrato, enquanto as variações de fosfato (MBB-P e $\mathrm{MBB}+\mathrm{P})$ resultaram em crescimento semelhante (Fig. 2). Os valores médios observados nos tratamentos envolvendo as interações de nitrato e fosfato $(\mathrm{MBB}+\mathrm{N} / \mathrm{P})$ foram inferiores em relação aos tratamentos dos nutrientes isolados, com exceção do tratamento MBB-N/P que praticamente igualou-se a MBB-N (Fig. 2).

A Anova entre os valores obtidos nos diferentes tratamentos de variação de nutrientes (Tab. 3) mostrou que os mesmos foram significativamente diferentes $(\mathrm{F}=13,26 ; \mathrm{p}<0,00) . \mathrm{O}$ teste de Tukey demonstrou que a produção de clorofila $a$ foi significativamente maior em MBB $(\mathrm{dms}=8,33)$ que em MBB-N, $\mathrm{MBB}+\mathrm{P}, \mathrm{MBB}-\mathrm{P}, \mathrm{MBB}+\mathrm{N} / \mathrm{P}$ e $\mathrm{MBB}-\mathrm{N} / \mathrm{P}$, divergindo apenas de $\mathrm{MBB}+\mathrm{N}$, onde, estatisticamente, não houve diferença significativa de MBB, mas diferiu de MBB-N $(\mathrm{dms}=8,33)$ por apresentar um crescimento mais significativo. Contudo, este mesmo teste mostrou que os tratamentos com nutrientes isolados $\mathrm{MBB}+\mathrm{N}$, $\mathrm{MBB}+\mathrm{P}$ e MBB-P apresentaram maior crescimento $(\mathrm{dms}=8,33)$ que nos tratamentos $\mathrm{MBB}-\mathrm{N} / \mathrm{P}$, $\mathrm{MBB}+\mathrm{N} / \mathrm{P}$ que mantinham variações dos nutrientes em conjunto.

\section{Discussão}

Aparentemente, devido à maior produção de clorofila $a$ ter sido observada por volta do $40^{\circ}$ dia de cultivo, nos tratamentos isolados de nitrato ou fosfato (com exceção de MBB-N), é possível inferir que a limitação do crescimento desta alga provavelmente ocorreu devido ao esgotamento destes nutrientes no meio de cultura. De acordo com Allan (1995), ambientes de água doce desprovidos de um fluxo unidirecional apresentam uma maior depleção de nutrientes, já que a correnteza resulta em uma contínua renovação destes nutrientes e de gases. Além disso, a

Tabela 3. Valores de clorofila $a \mathrm{em} \mu \mathrm{g} \mathrm{mL} \mathrm{m}^{-1}$ por tratamento expressando a biomassa de Schizomeris leibleinii Kützing em diferentes concentrações de nitrato e fosfato ao final de 50 dias $\left(n=25,{ }^{*} n=24\right)$. (ep = erro padrão; Mín = valor mínimo; Máx = valor máximo).

\begin{tabular}{|c|c|c|c|c|c|c|c|}
\hline & \multicolumn{7}{|c|}{ Meios de cultura } \\
\hline & $\mathrm{MBB}$ & MBB-N & $\mathrm{MBB}+\mathrm{N}$ & MBB-P* & $\mathrm{MBB}+\mathrm{P}$ & $\mathrm{MBB}+\mathrm{N} / \mathrm{P}$ & MBB-N/P \\
\hline Média & 26,01 & 9,17 & 20,06 & 15,62 & 13,94 & 4,84 & 9,43 \\
\hline ep & 3,87 & 0,90 & 1,86 & 1,48 & 1,98 & 0,84 & 1,0 \\
\hline Mín & 2,31 & 2,60 & 5,20 & 1,44 & 0,58 & 1,44 & 2,31 \\
\hline Máx & 70,23 & 18,79 & 37,86 & 28,32 & 32,08 & 16,18 & 21,39 \\
\hline
\end{tabular}




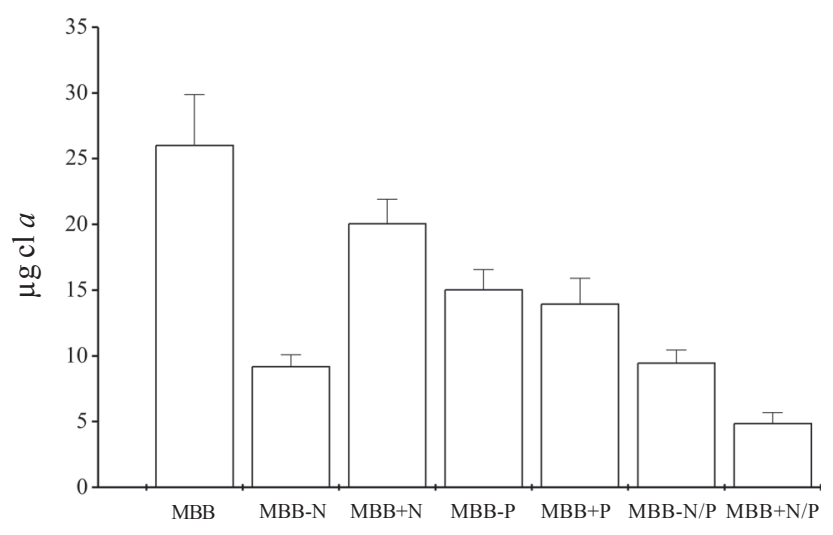

Figura 2. Variação média da biomassa (clorofila $a$ ) em Schizomeris leibleinii Kützing durante 50 dias de crescimento em diferentes concentrações de nitrato e fosfato em MBB.

provável interrupção dos ciclos do nitrogênio e do fósforo, bem como de outros elementos, podem ter comprometido o contínuo desenvolvimento algal (Margalef 1983).

Com exceção de $\mathrm{MBB}+\mathrm{N}$, como indicado pela não diferença das médias pelo teste de Tukey, cuja concentração de clorofila $a$ no $10^{\circ}$ dia esteve próxima do controle (MBB), a produtividade relativamente superior a MBB nos 10 primeiros dias de crescimento foi observada em MBB-N, MBB-P, e MBB-N/P (Tab. 2). Estes dados sugerem que, tanto nos tratamentos isolados ou em associação, o crescimento algal ocorreu de maneira mais rápida nestes tratamentos do que no controle e nos meios de cultura com maiores concentrações de nitrato e fosfato. Isto indica que Schizomeris leibleinii Kützing, em virtude da deficiência destes nutrientes isolados ou associados, disponibilizou boa parte de sua energia na reprodução como uma forma de sobreviver às condições tidas como adversas para a mesma. Sabe-se que mudanças súbitas no ambiente provocam, na maioria das vezes, uma esporulação abundante (Smith 1987). Além disso, McBride (1968) e Pereira \& Branco (2004), efetuando experimentos que envolviam a produção de zoósporos em S. leibleinii, observaram que esta alga esporulava copiosamente quando era transferida de um meio de cultura completo para outro meio menos concentrado (diluído com água destilada).

Analisando-se os gráficos de crescimento de $S$. leibleinii obtidos em $\mathrm{MBB}, \mathrm{MBB}+\mathrm{N}$ e $\mathrm{MBB}+\mathrm{P}$ (Fig. 1A, C, E), conclui-se que os mesmos são típicos de espécies estrategistas em $k$, mostrando inclusive, que sob altas concentrações de nitrato $(14,47 \mathrm{mM})$ e fosfato $(8,6 \mathrm{mM})$ esta espécie mantém um padrão de crescimento semelhante ao controle MBB, apesar da produtividade ser baixa. De acordo com Odum (1988) e Biggs et al. (1998) os estrategistas em $k$ apresentam um potencial de crescimento menor, pois dividem energia em favor de sua manutenção e desta maneira são capazes de obter sucesso competitivo em condições de alta densidade populacional. Biggs et al. (1998) estudaram várias espécies de algas e as classificaram em subgrupos de estrategistas em $k$, denominados $C$, $S$ e $R$. Os estrategistas pertencentes ao subgrupo $C$ são adaptados fisiologicamente a altas concentrações de nutrientes inorgânicos, pois vivem em hábitats eutrofizados, como acontece com S. leibleinii. Do mesmo modo, Biggs et al. (1998) apresentaram características consideradas adequadas a estrategistas $k$ (subgrupo $C$ ), que são encontradas com freqüência em S. leibleinii, como: i) formação de uma estrutura filamentosa com células médias a grandes, que compete melhor por nutrientes e luz, ii) sistema basal de fixação (p. ex. rizóides) que fornece grande resistência ao fluxo d'água, iii) capacidade de formar grandes biomassas e iv) freqüente reprodução por zoosporogênese ou fragmentação dos filamentos.

A avaliação global dos efeitos do nitrato e fosfato mostrou que quando o meio de cultura apresentava baixas concentrações de nitrato $(0,294 \mathrm{mM})$ e/ou fosfato $(0,172 \mathrm{mM})$ houve crescimento, mas inferior em relação ao controle (Fig. 2). Resposta semelhante foi observada por De Vries et al. (1985) em experimentos envolvendo os efeitos do nitrogênio e fósforo na produtividade de Stigeoclonium Kützing.

$O$ aumento na concentração de nitrato em $\mathrm{MBB}+\mathrm{N}(14,47 \mathrm{mM})$ aumentou a produtividade de Schizomeris leibleinii, no entanto, sem superar os valores observados em MBB. A redução de nitrato em MBB-N, por outro lado, provocou uma resposta negativa da alga a esta concentração (Fig. 2). Sob altas concentrações de fosfato, a resposta de crescimento foi inferior à resposta obtida em $\mathrm{MBB}+\mathrm{N}$. Contudo, $\mathrm{o}$ crescimento observado em MBB-N/P foi praticamente o mesmo de MBB-N, sugerindo que a alga é mais sensível às alterações nas concentrações de nitrogênio. Estes resultados indicam que o nitrogênio é o nutriente limitante à produção de biomassa em $S$. leibleinii, pois a alga respondeu com maior produtividade a altas concentrações de nitrato do que em altas concentrações de fosfato (Fig. 2). Diferentemente de Cladophora Kützing, que produziu extensa proliferação de biomassa apenas com $\mathrm{P}$ quase tanto quanto $\mathrm{N}, \mathrm{P}$ e $\mathrm{K}$ juntos em um experimento de enriquecimento das águas de um lago com formas orgânicas e inorgânicas N, P, e combinados de N, P e 
$\mathrm{K}$; mostrando, obviamente que o $\mathrm{N}$ não foi o nutriente limitante ao crescimento de Cladophora (Neil \& Owen 1964). De Vries et al. (1983) observaram efeitos semelhantes quanto ao crescimento verificado em S. leibleinii em análises feitas com onze linhagens de Stigeoclonium Kützing crescendo sob várias concentrações de nitrogênio e fósforo.

A produção de biomassa em Schizomeris leibleinii foi baixa em $\mathrm{MBB}+\mathrm{N} / \mathrm{P}$ quando comparada ao crescimento observado nos tratamento $\mathrm{MBB}+\mathrm{P}$ e $\mathrm{MBB}+\mathrm{N}$. Estes resultados sugerem que mesmo quando concentrações adequadas de nitrogênio são fornecidas, o excesso de fósforo torna-se um fator limitante, pois é possível supor que o mesmo possa estar influenciando negativamente a absorção de nitrogênio ou inibindo o seu aproveitamento pela alga.

As produtividades médias verificadas em $\mathrm{MBB}+\mathrm{P}$ e MBB-P foram muito semelhantes (Fig. 2) sugerindo que $S$. leibleinii tolera elevadas variações na concentração de fosfato $(0,172 \mathrm{mM}$ a $8,6 \mathrm{mM})$. Considerando-se que o fósforo freqüentemente limita a produtividade de plantas e outros autótrofos em ambientes aquáticos (Allan 1995), S. leibleinii mostrou grande tolerância às concentrações deste nutriente, concordando com Branco \& Pereira (2002) que encontraram correlação entre fósforo total e a biomassa desta alga em ambiente poluído.

Já com nitrato, a resposta de crescimento foi relativamente alta, mas limitada em $\mathrm{MBB}+\mathrm{N}$ $(14,47 \mathrm{mM})$ mostrando limitação na absorção desta forma de nitrogênio sob esta concentração. O crescimento algal obtido no controle foi maior quando comparado aos demais tratamentos (Fig. 2), sugerindo, assim, que as concentrações de nitrato $(2,94 \mathrm{mM}) \mathrm{e}$ fosfato $(1,72 \mathrm{mM})$ presentes em MBB podem ser consideradas mais favoráveis ao crescimento de S. leibleinii.

O nitrogênio, na forma de nitrato, mostrou ser o nutriente limitante ao crescimento desta clorofícea, que nas condições empregadas, revelou grande tolerância a altas concentrações deste nutriente. No entanto Branco \& Pereira (2002) encontraram correlação positiva entre $S$. leibleinii e a concentração de amônio $\left(\mathrm{NH}_{4}^{+}\right)$no ambiente. Bushong \& Bachmann (1989) observaram em alguns experimentos "in situ" envolvendo algas do perifíton, que a adição de amônio, e não nitrato, estimulava o crescimento do mesmo; todavia esta resposta não foi interpretada como limitação de nitrogênio, considerando que ambas as formas podem conter o nutriente limitante. De acordo com Syrett (1962) e Morris (1974), menos energia é necessária para assimilar amônio que nitrato, visto que o amônio está disponível para a utilização diretamente após a absorção, enquanto o nitrato deve primeiramente ser reduzido a amônio antes da utilização, processo este que requer substancial energia.

De acordo com os presentes resultados e com dados de literatura (Campbell \& Sarafis 1972; Sarma \& Chaudhary 1975; Yadava \& Pandey 1980; Necchi Júnior et al. 1994; Tiwari 1994; Branco \& Pereira 2002), pode-se considerar que S. leibleinii é um importante indicador biológico de poluição orgânica, pois apresenta crescimento acentuado em condições eutróficas.

Embora a espécie possa ter papel relevante na indicação biológica de poluição orgânica, o ideal para a avaliação ambiental é a informação fornecida pela comunidade (Biggs 1989; McCormick \& Cairns 1994; Kelly 1998; Kelly \& Whitton 1998; Vis et al. 1998). Neste sentido, o desenvolvimento de índices ecológicos específicos para ambientes tropicais deve ser estimulado (Kelly 1998). A inclusão de S. leibleinii deverá ser imprescindível para a produção de um índice baseado nas macroalgas, considerando-se sua importância e freqüente ocorrência em ambientes lóticos tropicais eutrofizados.

\section{Agradecimentos}

Agradecemos a Maria Helena Carabolante (DZB/ IBILCE/UNESP), pelo auxílio nas análises de laboratório.

\section{Referências bibliográficas}

Allan, J.D. 1995. Stream ecology: structure and function of running waters. New York, Chapman \& Hall.

Biggs, B.J.F. 1989. Biomonitoring of organic pollution using periphyton, South Branch, Canterbury, New Zealand. New Zealand Journal of Marine and Freshwater Research 23: 263-274.

Biggs, B.J.F.; Stevenson, R.J. \& Lowe, R.L. 1998. A habitat matrix conceptual model for stream periphyton. Archiv für Hydrobiologie 143: 21-56.

Branco, L.H.Z. \& Pereira, J.L. 2002. Evaluation of seasonal dynamics and bioindication potential of macroalgal communities in polluted tropical stream. Archiv für Hydrobiologie 155: 147-161.

Bushong, S.J. \& Bachman, R.W. 1989. In situ nutrient enrichment experiments with periphyton in agricultural streams. Hydrobiologia 178: 1-10.

Campbell, E.O. \& Sarafis, V. 1972. Schizomeris - a growth form of Stigeoclonium tenue (Chlorophyta: Chaetophoraceae). Journal of Phycology 8: 276-282. 
De Vries, P.J.R. \& Hotting, E.J. 1985. Bioassays with Stigeoclonium tenue Kütz. on waters receiving sewage effluents. Water Research 19: 1405-1410.

De Vries, P.J.R.; Torenbeek, M. \& Hillebrand, H. 1983. Bioassays with Stigeoclonium Kütz. (Chlorophyceae) to identify nitrogen and phosphorus limitations. Aquatic Botany 17: 95-106.

De Vries, P.J.R.; De Smet, S.J.M. \& Heide, J. van der. 1985. Effects of phosphorus and nitrogen enrichment on the yield of some strains Stigeoclonium Kütz. (Chlorophyceae). Freshwater Biology 15: 95-103.

Francke, J.A. \& Ten Cate, H.J. 1980. Ecotypic differentiation in response to nutritional factors in the algal genus Stigeoclonium Kütz. (Chlorophyceae). British Phycological Journal 15: 343-355.

Hillebrand, H. 1983. Development and dynamics of floating clusters of filamentous algae. Pp. 31-39. In: R.G. Wetzel (ed.). Periphyton in freshwater ecosystems. Hague, Junk Publishers.

Kelly, M.G. 1998. Use of community-based indices to monitor eutrophication in European rivers. Environmental Conservation 25: 22-29.

Kelly, M.G. \& Whitton, B.A. 1998. Biological monitoring of eutrophication in rivers. Hydrobiologia 38: 55-67.

Klapwijk, S.P.; De Boer, T.F. \& Rijs, M.J. 1983. Effect of agricultural wastewater on benthic algae in ditches in the Netherlands. Pp. 311-319. In: R.G. Wetzel (ed.). Periphyton in freshwater ecosystems. Hague, Junk Publishers.

Maloney, T.E. \& Miller, W.E. 1975. Algal assays: development and application of water quality parameters. Pp. 344-355. In: ASTM STP 573. [S.1.]. Philadelphia, American Society for Testing and Materials.

Margalef, R. 1983. Limnologia. Barcelona, Omega.

McBride, G.E. 1968. A classroom demonstration of zoospore production in the green alga Schizomeris leibleinii Kuetz. Journal of Phycology 4: 251-252.

McCormick, P.V. \& Cairns, J.Jr. 1994. Algae as indicators of environmental change. Journal of Applied Phycology 6: 509-526.

Morris, I. 1974. Nitrogen assimilation and protein synthesis. Pp. 583-609. In: W.D.P. Steward (ed.). Algal physiology and biochemistry. Berkeley, University of California Press.
Necchi Júnior, O.; Branco, L.H.Z. \& Dip, M.R. 1994. Uso de macroalgas para avaliação da poluição orgânica no Rio Preto, noroeste do estado de São Paulo. Anais da Academia Brasileira de Ciências 66: 359-371.

Neil, J.H. \& Owen, G.E. 1964. Distribution, environmental requirements, and significance of Cladophora in the Great Lakes. Proceedings of $7^{\text {th }}$ Conference on Great Lakes Research 11: 113-121.

Nichols, H.W. 1973. Growth media-freshwater. Pp.7-24. In: H.W. Nichols (ed.). Handbook of phycological methods: culture methods and growth measurements. Cambridge, Cambridge University Press.

Odum, E.P. 1988. Ecologia. Rio de Janeiro, Guanabara Koogan.

Pal, R.; Chatterjee, P. \& Das, T.M. 1992. Algological evaluation of organic pollution level of Hugli estuary, West Bengal, India. Phykos 31: 69-75.

Pereira, J.L. \& Branco, L.H.Z. 2004. Zoosporogênese: uma demonstração prática com a alga verde Schizomeris leibleinii (Chaetophorales, Chlorophyta). Revista Unorp 3: 7-13.

Sarma, Y.S.R.K. \& Chaudhary, B.R. 1975. On a new cytological race of Schizomeris leibleinii Kütz. Hydrobiologia 47: 181-191.

Smith, G.M. 1987. Botânica criptogâmica. v.1. Lisboa, Fundação Calouste Gulbenkian.

Syrett, P.J. 1962. Nitrogen assimilation. Pp. 171-188. In: R.A. Lewin (ed.). Physiology and biochemistry of algae. New York, Academic Press.

Tiwari, M.G. 1994. New record of Schizomeris leibleinii (alga) from polluted habitats of south Chotanagpur, Bihar. Journal of Ecobiology 6: 71-72.

Vis, C.; Hudon, C.; Cattaneo, A. \& Pinel-Alloul, B. 1998. Periphyton as an indicator of water quality in the St. Lawrence River (Québec, Canada). Environmental Pollution 101: 13-24.

Wetzel, R.G. \& Likens, G.E. 1990. Limnological analyses. New York, Springer-Verlag.

Wetzel, R.G. \& Westlake, D.F. 1971. Periphyton. Pp. 42-50. In: R.A. Vollenweider (ed.). A manual on methods for measuring primary production in aquatic environments. Oxford, Blackwell IBP Handbook 12.

Yadava, R.N. \& Pandey, D.C. 1980. Certain cultural observations on Schizomeris leibleinii Kuetz. Phykos 19: 204-209.

Zar, J.H. 1999. Biostatistical analysis. New Jersey, Prentice Hall. 